\title{
CONTRIBUTIONS TO THE THEORY OF HERMITIAN SERIES
}

\section{THE REPRESENTATION PROBLEM*}

BY

\section{EINAR HILLE}

1. Introduction. In the first note of this series [2] the author laid a broad foundation for the theory of Hermitian series in the complex domain. A number of questions encountered during this investigation were merely mentioned and detailed discussion had to be postponed till later communications. The representation or expansion problem was such a question, that is, the problem of finding necessary and sufficient conditions in order that an analytic function shall be representable by an Hermitian series for complex values of the variable. This problem is solved in the present note.

Let $H_{n}(z)$ denote the $n$th normalized orthogonal function of Hermite

$$
H_{n}(z)=\left[\pi^{1 / 2} 2^{n} n !\right]^{-1 / 2}(-1)^{n} e^{z^{2} / 2} \frac{d^{n}}{d z^{n}}\left[e^{-z^{2}}\right] .
$$

If $f(x)$ is a measurable function such that $x^{n} \exp \left[-x^{2} / 2\right] f(x) \in L_{1}(-\infty, \infty)$ for $n=0,1,2, \cdots$, then $f(x)$ has an associated Fourier-Hermite series

$$
f(x) \sim \sum_{n=0}^{\infty} f_{n} H_{n}(x)
$$

where

$$
f_{n}=\int_{-\infty}^{\infty} f(t) H_{n}(t) d t
$$

This series is ordinarily not convergent, but if we assume for instance that $\exp \left[-\alpha x^{2}\right] f(x) \varepsilon L_{1}(-\infty, \infty)$ for some $\alpha<1 / 2$, then the series (1.2) can be summed to the sum $f(x)$ for almost all $x$ by a generalization of the Abel method of summation. $\ddagger$ Under this assumption the series

$$
f(x ; s)=\sum_{n=0}^{\infty} f_{n} \boldsymbol{H}_{n}(x) s^{n}
$$

converges for

* Presented to the Society, October 28, 1939; received by the editors November 6, 1939.

$\dagger$ Numbers in square brackets refer to the references at the end of this paper.

$\ddagger$ See E. Hille [1, pp. 448-450, 453]. 


$$
|s|<\left(\frac{1-2 \alpha}{1+2 \alpha}\right)^{1 / 2} \equiv r_{\alpha} .
$$

Further $f(x ; s)$ is a holomorphic function of $s$ in the interior of the ellipse in the complex $s$-plane having its vertices at the points $s= \pm 1, \pm i r_{\alpha}$. As $s \rightarrow+1$ along the real axis, $f(x ; s) \rightarrow f(x)$ for almost all values of $x$. It follows in particular that if the series (1.2) converges in a set $E$ of positive measure, its sum equals $f(x)$ almost everywhere in $E$ provided $\exp \left[-\alpha x^{2}\right] f(x) \varepsilon L_{1}(-\infty, \infty)$ for some $\alpha<1 / 2$.

It may happen that the series (1.2) converges also for complex values of the variable. If so, it is known that the domain of absolute convergence is a strip $S_{\tau}:-\tau<y<\tau, z=x+i y$, where

$$
\tau=-\limsup _{n \rightarrow \infty}(2 n+1)^{-1 / 2} \log \left|f_{n}\right| .
$$

For a proof, see E. Hille [2, chap. 2] and G. Szegö [7, p. 246]. The series may converge on the lines of convergence $y= \pm \tau$, but it diverges outside of $S_{r}$. In this case the series

$$
\sum_{n=0}^{\infty} f_{n} H_{n}(z) \equiv f(z)
$$

defines an analytic function which is holomorphic in $S_{r}$.

Such a function $f(z)$ is of course characterized by the fact that its FourierHermite coefficients satisfy the inequality

$$
\left|f_{n}\right| \leqq M(\epsilon) \exp \left[-(\tau-\epsilon)(2 n+1)^{1 / 2}\right]
$$

for every positive $\epsilon$, but a direct characterization of $f(z)$ in terms of functiontheoretical properties would seem desirable. A complete solution of this problem is given by

THEOREM 1. Let $f(z)$ be an analytic function. A necessary and sufficient condition in order that the Fourier-Hermite series

$$
\sum_{n=0}^{\infty} f_{n} \boldsymbol{H}_{n}(z), \quad f_{n}=\int_{-\infty}^{\infty} f(t) \boldsymbol{H}_{n}(t) d t,
$$

shall exist and converge to the sum $f(z)$ in the strip $S_{\tau}:-\tau<y<\tau$, is that $f(z)$ is holomorphic in $S_{\tau}$ and that to every given $\beta, 0 \leqq \beta<\tau$, there exists a finite positive $B(\beta)$ such that

$$
|f(x+i y)| \leqq B(\beta) \exp \left[-|x|\left(\beta^{2}-y^{2}\right)^{1 / 2}\right]
$$

for $-\infty<x<\infty,-\beta \leqq y \leqq \beta$. 
The proof that this condition is necessary will be given in $\$ 2$. The sufficiency proof occurs in $\$ 5$ and is preceded by material relating to the asymptotic behavior of $H_{n}(z)$ for large values of $n$ based on the investigations of R. E. Langer [3]. An application to functions meromorphic in an upper or a lower half-plane is given in $\$ 6$.

As far as I know there have only been two previous contributions to the representation problem for Hermitian series, those of G. N. Watson [9, pp. 417-421] and O. Volk [8]. Their equivalent conditions require that $f(z)$ shall be holomorphic in $-\tau \leqq y \leqq \tau$ and that the function $g(z) \equiv z^{-1} \exp \left(z^{2} / 2\right) f(z)$ shall have the properties (i) $g(x+i y) \rightarrow 0$ as $|x| \rightarrow \infty,-\tau \leqq y \leqq \tau$, and (ii) $g(x \pm i \tau) \varepsilon L_{1}(-\infty, \infty)$. It is clear that these conditions while sufficient are far from necessary.

2. Proof of necessity. The reader will be supposed to have some knowledge of the properties of Hermitian polynomials and of solutions of the Weber-Hermite differential equations. For convenient summaries of the former theory see E. Hille [1] and G. Szegö [7], for the latter see Whittaker-Watson [12].

The only property which will be used in the present paragraph is the generating function*

$$
\begin{aligned}
\sum_{n=0}^{\infty} H_{n}(u) & H_{n}(v) s^{n} \\
= & \pi^{-1 / 2}\left(1-s^{2}\right)^{-1 / 2} \exp \left\{-\frac{\left(1+s^{2}\right)\left(u^{2}+v^{2}\right)-4 s u v}{2\left(1-s^{2}\right)}\right\} .
\end{aligned}
$$

The series converges for arbitrary complex values of $u$ and $v$ when $|s|<1$. Putting $u=z=x+i y, v=\bar{z}=x-i y$, we get

$$
\sum_{n=0}^{\infty}\left|H_{n}(z)\right|^{2} s^{n}=\pi^{-1 / 2}\left(1-s^{2}\right)^{-1 / 2} \exp \left\{-\frac{1-s}{1+s} x^{2}+\frac{1+s}{1-s} y^{2}\right\} .
$$

We shall also need information concerning integrals of the form

$$
J(p, q, \nu)=\int_{0}^{\infty} \exp \{-p t-q / t\} t^{-v} d t,
$$

where $p$ and $q$ are positive and $\nu=3 / 2,2$ or $5 / 2$. Now

$$
J(p, q, 3 / 2)=\pi^{1 / 2} q^{-1 / 2} \exp \left[-2(p q)^{1 / 2}\right],
$$

as is easily verified. Since $J(p, q, 5 / 2)=-J_{q}^{\prime}(p, q, 3 / 2)$,

$$
J(p, q, 5 / 2)=\pi^{1 / 2} q^{-3 / 2}\left[1 / 2+(p q)^{1 / 2}\right] \exp \left[-2(p q)^{1 / 2}\right] .
$$

\footnotetext{
* See, for instance, E. Hille [1, pp. 439-440].
} 
Finally, by Schwarz' inequality

$$
J(p, q, 2) \leqq \pi^{1 / 2} q^{-1}\left[1 / 2+(p q)^{1 / 2}\right]^{1 / 2} \exp \left[-2(p q)^{1 / 2}\right] .
$$

Suppose now that the series (1.6) exists and converges to $f(z)$ in the strip $-\tau<y<\tau$. It follows immediately that $f(z)$ is holomorphic in the strip and it remains merely to estimate $f(z)$. Let $\beta$ be given, $0<\beta<\tau$, and put $\alpha=(\beta+\tau) / 2$. In view of $(1.5)$, the series

$$
\sum_{n=0}^{\infty}\left|f_{n}\right|^{2} \exp (2 \alpha N) \equiv A^{2}(\beta)
$$

is convergent. Here and in the following we shall write

$$
N=(2 n+1)^{1 / 2} \text {. }
$$

Let us put

$$
H(x, y ; \alpha)=\sum_{n=0}^{\infty}\left|H_{n}(x+i y)\right|^{2} \exp (-2 \alpha N) .
$$

Cauchy's inequality then gives

$$
|f(x+i y)| \leqq A(\beta)[H(x, y ; \alpha)]^{1 / 2} .
$$

Let us first obtain $H(x, y ; \alpha)$ in closed form. Forming

$$
\sum_{n=0}^{\infty}\left|H_{n}(z)\right|^{2} J\left(2 n+1, \alpha^{2}, 3 / 2\right)
$$

and using (2.2) and (2.3) we get after some simplifications

$$
\begin{aligned}
H(x, y ; \alpha)=\frac{\alpha}{\pi} \int_{0}^{\infty}\left(1-e^{-4 t}\right)^{-1 / 2} \exp \left\{-\alpha^{2} / t-t-\right. & x^{2} \tanh t \\
& \left.+y^{2} \operatorname{coth} t\right\} t^{-3 / 2} d t
\end{aligned}
$$

where $\tanh t$ denotes the hyperbolic tangent of $t$.

Elementary considerations show that

$$
\operatorname{coth} t \leqq(1+t) / t, \quad-\tanh t \leqq-t /(1+t), \quad t>0 .
$$

Substituting these dominants for the hyperbolic functions in (2.10) we obtain $(\pi / \alpha) H(x, y ; \alpha) \leqq H_{0}(x, y ; \alpha)$

$$
\begin{aligned}
& \equiv e^{y^{2}} \int_{0}^{\infty}\left(1-e^{-4 t}\right)^{-1 / 2} \exp \left\{-\frac{\alpha^{2}-y^{2}}{t}-t-\frac{x^{2} t}{1+t}\right\} t^{-3 / 2} d t \\
& \equiv e^{y^{2}} H_{1}(x, y ; \alpha),
\end{aligned}
$$

and it remains to estimate $H_{1}(x, y ; \alpha)$. 
Let $\xi=4 \alpha|x|^{-1}$ and put

$$
H_{1}=H_{11}+H_{12}, H_{11}=\int_{0}^{\xi} \cdots, H_{12}=\int_{\xi}^{\infty} \cdots
$$

with obvious notation.

We note that $t /(1+t)$ is an increasing function. Hence its least value in the interval $(\xi, \infty)$ is taken on at $t=\xi$. The inequality $1+u \leqq e^{u}$ gives

$$
(1+u)^{-1} \geqq e^{-u}, \quad\left(1-e^{-u}\right)^{-1} \leqq 1 / u+1
$$

and finally

$$
\left(1-e^{-4 t}\right)^{-1 / 2} \leqq(1 /(4 t)+1)^{1 / 2} \leqq t^{-1 / 2} / 2+1
$$

Hence

$$
H_{12} \leqq \exp \left[-\frac{4 \alpha x^{2}}{4 \alpha+|x|}\right]\left\{J\left(1, \alpha^{2}-y^{2}, 3 / 2\right)+(1 / 2) J\left(1, \alpha^{2}-y^{2}, 2\right)\right\} .
$$

Supposing $|x| \geqq 4 \alpha$, which is no essential restriction, and using (2.3) and (2.5), we get

$$
\begin{aligned}
H_{12} \leqq \pi^{1 / 2} \exp \left[-2 \alpha|x|-2\left(\alpha^{2}-y^{2}\right)^{1 / 2}\right]\left(\alpha^{2}-y^{2}\right)^{-1} \\
\cdot\left\{(1 / 2)\left[1 / 2+\left(\alpha^{2}-y^{2}\right)^{1 / 2}\right]^{1 / 2}+\left(\alpha^{2}-y^{2}\right)^{1 / 2}\right\} .
\end{aligned}
$$

If $-\beta \leqq y \leqq \beta$, the right-hand side does not exceed

$$
\frac{10 \beta+7}{(\tau+3 \beta)(\tau-\beta)} \exp [-2 \alpha|x|]
$$

that is,

$$
H_{12}(x, y ; \alpha) \leqq B_{1}(\beta) \exp [-2 \alpha|x|]
$$

for $|x| \geqq 4 \alpha,-\beta \leqq y \leqq \beta$, and by a suitable modification of $B_{1}(\beta)$ we can of course gain that the inequality is true for all $x$. Here and in the following $B(\beta)$, with or without subscripts, denotes a monotone increasing function of $\beta$, defined and continuous for $0 \leqq \beta<\tau$, but conceivably tending to infinity as $\beta \rightarrow \tau$.

For $t>0$ we have

$$
-t /(1+t)=-t+t^{2} /(1+t)<-t+t^{2} .
$$

Further, for $0<t \leqq \xi \leqq 1$, that is, for $|x| \geqq 4 \alpha$,

$$
\left(1-e^{-4 t}\right)^{-1 / 2} \leqq\left(1-e^{-4}\right)^{-1 / 2} t^{-1 / 2}<2 t^{-1 / 2} .
$$

Hence 


$$
\begin{aligned}
& H_{11}<2 e^{\xi^{2} x^{2}} \int_{0}^{\xi} \exp \left\{-\left(\alpha^{2}-y^{2}\right) / t-x^{2} t\right\} t^{-2} d t \\
& <2 e^{16 \alpha^{2}} J\left(x^{2}, \alpha^{2}-y^{2}, 2\right) \\
& <2 \pi^{1 / 2} e^{16 \tau^{2}}\left(\alpha^{2}-y^{2}\right)^{-1}\left[1 / 2+|x|\left(\alpha^{2}-y^{2}\right)^{1 / 2}\right]^{1 / 2} \exp \left[-2|x|\left(\alpha^{2}-y^{2}\right)^{1 / 2}\right] \\
& <4 e^{16 \tau^{2}}\left(\alpha^{2}-y^{2}\right)^{-1}\left[1+|x|^{1 / 2}\left(\alpha^{2}-y^{2}\right)^{1 / 4}\right] \exp \left[-2|x|\left(\alpha^{2}-y^{2}\right)^{1 / 2}\right] .
\end{aligned}
$$

Suppose again that $|y| \leqq \beta$. Then $\left(\beta^{2}-y^{2}\right)^{1 / 2}<\left(\alpha^{2}-y^{2}\right)^{1 / 2}$ and the absolute maximum of

$$
|x|^{1 / 2}\left(\alpha^{2}-y^{2}\right)^{1 / 4} \exp \left\{-2|x|\left[\left(\alpha^{2}-y^{2}\right)^{1 / 2}-\left(\beta^{2}-y^{2}\right)^{1 / 2}\right]\right\}
$$

for all values of $x$ equals

Consequently

$$
\frac{1}{2}\left\{\frac{\alpha}{e(\alpha-\beta)}\right\}^{1 / 2} .
$$

$$
H_{11}<4 e^{16 \tau^{2}}\left(\alpha^{2}-\beta^{2}\right)^{-1}\left[1+\alpha^{1 / 2}(\alpha-\beta)^{-1 / 2}\right] \exp \left[-2|x|\left(\beta^{2}-y^{2}\right)^{1 / 2}\right]
$$

or

$$
H_{11}(x, y ; \alpha)<B_{2}(\beta) \exp \left[-2|x|\left(\beta^{2}-y^{2}\right)^{1 / 2}\right]
$$

for $-\beta \leqq y \leqq \beta$ and all values of $x$, since clearly the restriction $|x| \geqq 4 \alpha$ can be removed.

Combining the inequalities in (2.9) to (2.14) we get the desired estimate

$$
|f(x+i y)|<B(\beta) \exp \left[-|x|\left(\beta^{2}-y^{2}\right)^{1 / 2}\right],
$$

valid for every $z$ in the strip $-\beta \leqq y \leqq \beta$ and for every $\beta<\tau$. This completes the proof of the necessity of condition (1.7).

3 . Some asymptotic formulas. The sufficiency proof requires accurate information concerning the behavior of $H_{n}(z)$ for large values of $n$ when the distance of $z$ from the line segment $(-N, N)$ remains bounded. This question will be studied in this and the next following sections.

The Weber-Hermite differential equation

$$
w^{\prime \prime}+\left(2 \kappa+1-z^{2}\right) w=0,
$$

where $\kappa$ is an arbitrary real or complex parameter, has a solution $h_{\kappa}(z)$ which is uniquely characterized by the asymptotic property

$$
\lim e^{z^{2} / 2}(2 z)^{-\kappa} h_{\kappa}(z)=1
$$

when $|z| \rightarrow \infty$ and $|\arg z|<3 \pi / 4$. Here the power has its principal determination when $z$ and $\kappa$ are real positive. In the notation of Whittaker and Watson

$$
h_{\kappa}(z)=2^{\kappa / 2} D_{\kappa}\left(2^{1 / 2} z\right) \text {. }
$$


Since $H_{n}(z)$ satisfies (3.1) when $\kappa=n$ and

$$
H_{n}(z)=\left(\pi^{1 / 2} 2^{n} n !\right)^{-1 / 2} e^{-z^{2} / 2}\left[(2 z)^{n}+\cdots\right],
$$

we conclude that

$$
H_{n}(z)=\left(\pi^{1 / 2} 2^{n} n !\right)^{-1 / 2} h_{n}(z) .
$$

It was shown by Whittaker [11] that $h_{-\kappa-1}(i z)$ and $h_{-\kappa-1}(-i z)$ are also solutions of (3.1) and Watson [9, p. 395] proved that

$$
h_{\kappa}(z)=\pi^{-1 / 2} \Gamma(\kappa+1) 2^{\kappa}\left\{e^{\kappa \pi i / 2} h_{-\kappa-1}(i z)+e^{-\kappa \pi i / 2} h_{-\kappa-1}(-i z)\right\} .
$$

The asymptotic behavior of $h_{-\kappa-1}(-i z)$ was studied by Watson on two occasions. The result of his earlier paper [9, p. 416] is not good enough for our purposes, but that of his second paper [10, p. 142] more than meets our needs. Watson's analysis is based upon special representations of the functions involved by means of definite integrals to which he applies the method of steepest descent.

I prefer a different approach to the problem based upon the work of Langer [3] on the asymptotic behavior of solutions of certain classes of second order linear differential equations for large values of a parameter. This is a much more general approach and brings out the reason why different expansions are to be expected in different parts of the plane-the so-called phenomenon of Stokes. N. Schwid [6] has proved that Langer's method applies to the Weber-Hermite equation. Schwid does not discuss $h_{-\kappa-1}( \pm i z)$ explicitly, however, and in that part of the plane where we require information his results are not valid.* It is not necessary to give a detailed presentation of Langer's method. We shall merely state the results and refer to Langer and Schwid for all details.

In the following, $\kappa$ shall be real positive. We write

$$
\mathrm{K}=(2 \kappa+1)^{1 / 2}
$$

and $\dagger$

$$
\xi(z)=\int_{\mathrm{K}}^{z}\left[\mathrm{~K}^{2}-t^{2}\right]^{1 / 2} d t
$$

* His estimate of the order of the remainder term in formula (26), p. 353, is valid for bounded values of $z$ only.

† Strictly speaking we should take $-K$ as the lower limit of the integral when $z$ is in the left half-plane. Since $\xi(-K)$ is a real quantity, the imaginary part of $\xi(z)$ is not affected by this change and the only place where it matters is in the remainder terms of formulas (3.7), (3.9), and (3.11) below. Here we should write $\xi-\xi(-K)$ instead of $\xi$ in the left half-plane. The correction is of no importance, however, except in a small neighborhood of $z=-\mathrm{K}$ which is excluded from consideration anyway. 
The Langer theory shows that for any value of $z \neq \pm \mathrm{K}$ there are two linearly independent local solutions of (3.1) which for large values of $\kappa$ are of the form

$$
\left[\xi^{\prime}(z)\right]^{-1 / 2} \exp [ \pm i \xi(z)]\{1+O(1 / \xi)+O(1 / \kappa)\}
$$

and with the aid of which any solution can be expressed linearly. The coefficients will of course depend upon the determination chosen for the infinitely many-valued function $\xi(z)$. In the present case the situation is simplified by the fact that the imaginary part of $\xi(z)$ is only two-valued and the arcs $\Im[\xi(z)]=0$ are independent of the determination of $\xi(z)$. These arcs form a configuration symmetric with respect to the coordinate axes and divide the plane into four sectors $S_{j}$. The sector $S_{2}$, of main importance to us, is bounded by the line segment $(-K, K)$ and two transcendental curves $\Gamma_{1}$ and $\Gamma_{2}$ going from $\pm K$ to $\infty$. The angle of inclination of the tangent of $\Gamma_{1}$ with the positive real axis starts with the value $\pi / 3$ at $z=\mathrm{K}$ and tends to $\pi / 4$ as $|z| \rightarrow \infty$.

Suppose the determination of $\xi(z)$ to be chosen at some point $z_{0}$ in $S_{2}$ and let a particular solution $w(z)$ of (3.1) be expressed linearly in terms of the two local solutions (3.7) in some neighborhood of $z=z_{0}$. Both solutions can be continued analytically in $S_{2}$, the continuations agreeing everywhere with the local fundamental system. It follows that the expression of $w(z)$ remains valid at all interior points of $S_{2}$. It is only when we cross the boundary of $S_{2}$ that the situation may change and a different substitution may have to be used to express $w(z)$ in terms of the local fundamental system. Thus to every sector $S_{j}$ and to every given solution $w(z)$ there are corresponding coefficients $C_{j k}, j=1,2,3,4 ; k=1,2$, joining $w(z)$ with the fundamental system in $S_{j}$.

After these generalities let us choose $\xi(z)$ so that on the positive imaginary axis we have

$$
\begin{aligned}
\xi(i y)=-\pi \mathrm{K}^{2} / 4+(i / 2)\left\{y\left(\mathrm{~K}^{2}+y^{2}\right)^{1 / 2}\right. & \\
& \left.+\mathrm{K}^{2} \log \left[y / \mathrm{K}+\left(1+y^{2} / \mathrm{K}^{2}\right)^{1 / 2}\right]\right\},
\end{aligned}
$$

where the square roots and the logarithm have their principal values. We can then take as the fundamental system in $S_{2}$ two solutions $w_{2, k}(z)$ which for large values of $\kappa$ have the form

$$
w_{2, k}(z)=\left\{1-z^{2} / K^{2}\right\}^{-1 / 4} \exp [ \pm i \xi(z)]\{1+O(1 / \xi)+O(1 / \kappa)\}
$$

Here the plus sign goes with $k=1$ and the minus sign with $k=2$ and the fourth root is real positive on the positive imaginary axis. The parameter $\kappa$ is supposed to be large and this will make $|\xi(z)|$ large if we omit small neighborhoods of the points $z= \pm \mathrm{K}$ of radius $O\left(\kappa^{-1 / 6}\right)$ inside of which (3.9) is not valid.*

\footnotetext{
* Compare the second footnote on page 86 .
} 
We have consequently

$$
h_{-\kappa-1}(-i z)=A(\kappa) w_{2,1}(z)+B(\kappa) w_{2,2}(z)
$$

in $S_{2}$. In order to determine $A(\kappa)$ and $B(\kappa)$ we use (3.2). Multiply both sides of (3.10) by $e^{-z^{2} / 2}(-2 i z)^{1+\kappa}$ and let $z \rightarrow \infty$, say along the positive imaginary axis. The left-hand side tends to the limit 1 , whereas the right-hand side tends to a limit if and only if $B(\kappa)=0$. Setting $B(\kappa)=0$ and using (3.8) and (3.9), we can compute the value of $A(\kappa)$. The result is

$$
\begin{aligned}
h_{-\kappa-1}(-i z)=2^{-1 / 2} \mathrm{~K}^{-\kappa-1} \exp \left[(1 / 4) \mathrm{K}^{2}(1+\pi i)\right] \\
\cdot\left\{1-z^{2} / \mathrm{K}^{2}\right\}^{-1 / 4} \exp [i \xi(z)]\{1+O(1 / \xi)+O(1 / \kappa)\},
\end{aligned}
$$

valid in $S_{2}$ except in neighborhoods of $z= \pm \mathrm{K}$ of radius $O\left(\kappa^{-1 / 6}\right)$. This is the required basic asymptotic formula. It can be shown to be in agreement with Watson's formula quoted above.

4. Estimates of the imaginary part of $\xi(z)$. We shall need a couple of lemmas for the sufficiency proof.

Lemma 1. Let $\xi(x)$ be defined by (3.6) and let $-\mathrm{K}<x<\mathrm{K}, 0 \leqq y \leqq \mathrm{~K}$. Then

$$
\begin{aligned}
\Im[\xi(x+i y)]= & y\left(\mathrm{~K}^{2}-x^{2}\right)^{1 / 2}+(1 / 6) y^{3}\left(\mathrm{~K}^{2}-x^{2}\right)^{-1 / 2} \\
& +\rho \mathrm{K}^{2} y^{3}\left(\mathrm{~K}^{2}-x^{2}\right)^{-3 / 2},
\end{aligned}
$$

where $|\rho| \leqq 5 / 24$.

Proof. We have

$$
\begin{aligned}
\Im[\xi(x+i y)] & =\Im\left\{\int_{x}^{x+i y}\left(\mathrm{~K}^{2}-t^{2}\right)^{1 / 2} d t\right\} \\
& =\Re\left\{\int_{0}^{y}\left[\mathrm{~K}^{2}-x^{2}-2 i x v+v^{2}\right]^{1 / 2} d v\right\},
\end{aligned}
$$

where the value of the square root is real positive when $v=0$. But by elementary transformations

$$
\left(a^{2}+b\right)^{1 / 2}-a-\frac{b}{2 a}=-\frac{b^{2}}{4 a^{2}}\left[\left(a^{2}+b\right)^{1 / 2}+a+\frac{b}{2 a}\right]^{-1} .
$$

Substituting $a^{2}=\mathrm{K}^{2}-x^{2}, b=-2 i x v+v^{2}$, we find that the right-hand side becomes equal to $P / Q$ where

$$
\begin{aligned}
P & =v^{2}(v-2 i x)^{2}, \\
Q & =4\left(\mathrm{~K}^{2}-x^{2}\right)\left\{\left[\mathrm{K}^{2}-x^{2}-2 i x v\right.\right. \\
& \left.+v^{2}\right]^{1 / 2}+\left(\mathrm{K}^{2}-x^{2}\right)^{1 / 2} \\
& \left.+\left(v^{2}-2 i x v\right)\left(\mathrm{K}^{2}-x^{2}\right)^{-1 / 2}\right\} .
\end{aligned}
$$


Here $0 \leqq v \leqq y \leqq K$. The least value of $|Q|$ is obtained for $v=0$. On the other hand, $|v-2 i x|^{2} \leqq 5 \mathrm{~K}^{2}$. Hence $|P / Q|$ does not exceed

$$
(5 / 8) \mathrm{K}^{2}\left(\mathrm{~K}^{2}-x^{2}\right)^{-3 / 2} v^{2},
$$

the integral of which from $v=0$ to $v=y$ equals

$$
(5 / 24) \mathrm{K}^{2}\left(\mathrm{~K}^{2}-x^{2}\right)^{-3 / 2} y^{3} .
$$

This is the remainder term of formula (4.1). The other two terms are the result of substituting $a^{2}=\mathrm{K}^{2}-x^{2}, b=-2 i x v+v^{2}$ into $a+b /(2 a)$, integrating with respect to $v$ from 0 to $y$ and finally taking the real part.

LEMMA 2. For a fixed $x$, where $-\mathrm{K}<x<\mathrm{K}$, the maximum value of

$$
\Im[\xi(x+i y)]+|x|\left(\beta^{2}-y^{2}\right)^{1 / 2},
$$

when $0 \leqq y \leqq \beta$, is not less than

$$
\beta \mathrm{K}+\frac{\beta^{3}}{6 \mathrm{~K}^{3}}\left(\mathrm{~K}^{2}-x^{2}\right)-\frac{5}{24} \frac{\beta^{3}}{\mathrm{~K}} .
$$

In particular, this value is reached or exceeded on the ellipse

$$
E(\mathrm{~K}, \beta): \beta^{2} x^{2}+\mathrm{K}^{2} y^{2}=\beta^{2} \mathrm{~K}^{2} .
$$

Proof. By Lemma 1

$$
\begin{array}{r}
\Im[\xi(x+i y)] \geqq y\left(\mathrm{~K}^{2}-x^{2}\right)^{1 / 2}+(1 / 6) y^{3}\left(\mathrm{~K}^{2}-x^{2}\right)^{-1 / 2} \\
-(5 / 24) \mathrm{K}^{2} y^{3}\left(\mathrm{~K}^{2}-x^{2}\right)^{-3 / 2} .
\end{array}
$$

The maximum value of

$$
y\left(\mathrm{~K}^{2}-x^{2}\right)^{1 / 2}+|x|\left(\beta^{2}-y^{2}\right)^{1 / 2}
$$

for a fixed $x$ is reached when $y=(\beta / \mathrm{K})\left(\mathrm{K}^{2}-x^{2}\right)^{1 / 2}$, that is, on the ellipse $E(\mathrm{~K}, \beta)$, and equals $\beta \mathrm{K}$. Substitution of this value of $y$ gives formula (4.3).

5. The sufficiency proof. After all these preparations we can attack the sufficiency proof. It is assumed that $f(z)$ is holomorphic in the strip $S_{\tau}$ : $-\tau<y<\tau$, and that to every $\beta, 0<\beta<\tau$, there exists a finite positive $B(\beta)$ for which condition (1.7) holds.* This condition is evidently sufficient to ensure the existence of the Fourier-Hermite coefficients $f_{n}$ defined by the second half of formula (1.6). Thus the Fourier-Hermite series of $f(z)$ has a sense, and condition (1.7) also ensures that this series is Abel summable to the sum $f(x)$ everywhere on the real axis. In order to complete the proof of the theorem it

* It is of course only values of $\beta$ close to $\tau$ which are of interest, but it is no restriction to assume that $B(\beta)$ satisfies the conventions of $\S 2$ for all $\beta, 0 \leqq \beta<\tau$. The same conventions can be imposed on all $B$-functions introduced below. 
is then enough to prove that the series is convergent in the strip $S_{\tau}$, and this will be proved if we are able to show the existence, for every $\beta, 0<\beta<\tau$, of a finite positive $B^{*}(\beta)$ such that

$$
\left|f_{n}\right|<B^{*}(\beta) e^{-\beta N}, \quad \quad N=(2 n+1)^{1 / 2},
$$

for all $n$.

It is known that there exists a constant $A$ such that $\dagger$

$$
\left|H_{n}(t)\right| \leqq A
$$

for all integers $n$ and all real values of $t$. This inequality shows that

$$
\begin{aligned}
\left\{\int_{-\infty}^{-N+1}+\int_{N-1}^{\infty}\right\}|f(t)|\left|H_{n}(t)\right| d t & \leqq 2 A B(\beta) \int_{N-1}^{\infty} e^{-\beta t} d t \\
& <B_{1}(\beta) e^{-\beta N}
\end{aligned}
$$

On the interval $(-N+1, N-1)$ we use formula (3.4) obtaining

$$
\begin{aligned}
\int_{-N+1}^{N-1} f(t) H_{n}(t) d t= & \pi^{-3 / 4}\left(2^{n} n !\right)^{1 / 2}\left\{i^{n} \int_{-N+1}^{N-1} f(t) h_{-n-1}(i t) d t\right. \\
& \left.+(-i)^{n} \int_{-N+1}^{N+1} f(t) h_{-n-1}(-i t) d t\right\} .
\end{aligned}
$$

In these integrals we can choose the path of integration joining $-N+1$ with $N-1$ in an arbitrary manner provided it stays in the strip $S_{\tau}$. Moreover, we can choose a different path for each of the integrals involved. Let $E(N, \beta)$ be the ellipse of Lemma 2 and denote the points of intersection of the ellipse with the lines $x= \pm(N-1)$ by $P, Q, R$, and $S$, where $Q$ is in the first and $P$ in the second quadrant.

The path of integration $C_{2}$ for the integral involving $h_{-n-1}(-i t)$ shall consist of the line segments joining $-N+1$ with $P$ and $N-1$ with $Q$ connected by the arc $P Q$ of the ellipse. The path of integration $C_{1}$ for the integral involving $h_{-n-1}(i t)$ will be the image of $C_{2}$ in the real axis. Let us put

$$
J_{2}=\int_{C_{2}}|f(z)|\left|h_{-n-1}(-i z)\right||d z| .
$$

Along $C_{2}$ we are at a safe distance from the critical points $z= \pm N$ so that formula (3.11) is valid, if we replace $\kappa$ and $\mathrm{K}$ by $n$ and $N$ respectively. It follows that there exists a constant $M$ independent of $z$ and $n$ such that on $E(N, \beta)$ we have

† See E. Hille [1, pp. 435-436] and G. Szegö [7, p. 236]. 


$$
\left|h_{-n-1}(-i z)\right|<M A_{n}\left|1-z^{2} / N^{2}\right|^{-1 / 4} \exp \{-\Im[\xi(z)]\},
$$

where

$$
A_{n}=N^{-n-1} e^{n / 2}<B\left(2^{n} n !\right)^{-1 / 2} n^{-1 / 4},
$$

and $B$ is an absolute constant.

On the vertical parts of $C_{2}$ it is enough to know that $\Im[\xi(z)]>0$. The length of the vertical parts is $O\left(n^{-3 / 4}\right)$ and the factor $\left|1-z^{2} / N^{2}\right|^{-1 / 4}$ amounts at most to $O\left(n^{1 / 8}\right)$. Their contributions to $J_{2}$ are consequently of the form

$$
B_{2}(\beta)\left[2^{n} n !\right]^{-1 / 2} n^{-7 / 8} e^{-\beta N} .
$$

On the arc $P Q$ of the ellipse, Lemma 2 shows that

$$
\begin{aligned}
\int_{P}^{Q}|f(z)|\left|h_{-n-1}(z)\right||d z| & <B B(\beta)\left[2^{n} n !\right]^{-1 / 2} n^{-1 / 4} \\
\cdot \int_{P}^{Q} \exp \{-|x| & \left.\left(\beta^{2}-y^{2}\right)^{1 / 2}-\Im[\xi(x+i y)]\right\}\left|1-z^{2} / N^{2}\right|^{-1 / 4}|d z| \\
& <B_{3}(\beta)\left[2^{n} n !\right]^{-1 / 2} n^{-1 / 4} e^{-\beta N} \int_{P}^{Q}\left|1-z^{2} / N^{2}\right|^{-1 / 4}|d z| .
\end{aligned}
$$

The integral being $O(N)$, this expression is of the form

$$
B_{4}(\beta)\left[2^{n} n !\right]^{-1 / 2} n^{1 / 4} e^{-\beta N} .
$$

Similar estimates hold on $C_{1}$. We have then

$$
\left|\int_{-N+1}^{N-1} f(t) H_{n}(t) d t\right|<B_{5}(\beta) n^{1 / 4} e^{-\beta N},
$$

and combining (5.3) and (5.10) we get finally

$$
\left|f_{n}\right|<B_{6}(\beta) n^{1 / 4} e^{-\beta N} \text {. }
$$

This being true for every $\beta<\tau$, it is clear that the estimate implies the validity of (5.1). This completes the proof of Theorem 1.

6. Functions holomorphic or meromorphic in a half-plane. Suppose that $f(z)$ is holomorphic in the half-plane $y>-\alpha, \alpha>0$, and that $f(z)$ admits of a formal Fourier-Hermite series

$$
f(z) \sim \sum_{n=0}^{\infty} f_{n} H_{n}(z), \quad f_{n}=\int_{-\infty}^{\infty} f(t) H_{n}(t) d t .
$$

We shall show that if the series has a strip of convergence, then $f(z)$ cannot be of exponential type in the half-plane $y \geqq 0$. In other words, the existence of a 
strip of convergence implies a lower limit on the rate of growth of the function in its supposed half-plane of holomorphism. The precise statement is as follows.

THEOREм 2. Let $f(z)$ be holomorphic in the half-plane $y>-\alpha, \alpha>0$. Further let

$$
\limsup _{r \rightarrow \infty}(1 / r) \log \left|f\left(r e^{i \theta}\right)\right|=h(\theta) \leqq M, \quad 0 \leqq \theta \leqq \pi .
$$

The Fourier-Hermite expansion of $f(z)$ can never converge outside of the real axis.*

Proof. For the proof we need the following

Lемма 3. If $f(z)$ is holomorphic in the half-plane $y \geqq 0$ and if $\log |f(x)|<$ $-\beta|x|$, where $\beta>0$, and $\log \left|f\left(r e^{i \theta}\right)\right| \leqq M r$ when $0 \leqq \theta \leqq \kappa$, then $f(z) \equiv 0$.

This Lemma is implicitly contained in some results of F. Nevanlinna [4, pp. 11-12] and is explicitly stated by F. and R. Nevanlinna [5, p. 38] as a special case of a more general theorem. It is easily proved by forming the Laplace transform of $f(z)$ and rotating the line of integration through an angle of $\pi$ in the upper half-plane.

This Lemma applies directly to the present situation since the assumption that the Fourier-Hermite series of $f(z)$ has a strip of convergence implies the inequality $\log |f(x)|<-\beta|x|$ for some positive $\beta$ by virtue of Theorem 1 and this combined with the inequality (6.2) would force $f(z)$ to vanish identically.

We see in particular that the Fourier-Hermite series of an entire function of order $\rho$ will never converge outside of the real axis unless $\rho>1$ or $\rho=1$ and the function is of the maximal type. Some further results of F. and R. Nevanlinna [5, pp. 40-42] throw light upon the possible width of the strip of convergence of the Fourier-Hermite series of an entire function of order one and particular maximal type. We state the result for functions meromorphic in a half-plane.

THEOREM 3. Let $f(z)$ be meromorphic in the half-plane $y>-\alpha, \alpha>0$. Let its zeros and poles in the half-plane $y>-\alpha$ be $a_{1}, a_{2}, a_{3}, \cdots$ and $b_{1}, b_{2}, b_{3}, \cdots r e$ spectively, where in addition $\Im\left(b_{n}\right) \geqq \beta>0$. Put $\arg a_{n}=\alpha_{n}$, arg $b_{n}=\beta_{n}$, and let

$$
d=\limsup _{r \rightarrow \infty} \frac{1}{r}[b(r)-a(r)],
$$

where

$$
a(r)=\sum \sin \alpha_{n}, \quad b(r)=\sum \sin \beta_{n}
$$

* The same conclusion is valid if the hypotheses refer to a lower half-plane instead. This remark also applies to Theorem 3 below. 
and the summations extend over all zeros and poles respectively of absolute value between $\beta$ and $r$. Finally let

$$
q=\limsup _{r \rightarrow \infty}[r \log r]^{-1} \int_{0}^{\pi} \log \left|f\left(r e^{i \theta}\right)\right| \sin \theta d \theta .
$$

If $f(z)$ has a Fourier-Hermite series whose ordinate of convergence equals $\tau$, then $\tau \leqq \min (\alpha, \beta, q+\pi d)$.

In order to connect this theorem with the Nevanlinna theory we have merely to observe that by Theorem 1

$$
\limsup _{r \rightarrow \infty} \frac{1}{2 r}\{\log |f(-r)|+\log |f(r)|\} \leqq-\tau
$$

and substitution in the inequality of $\mathrm{F}$. and R. Nevanlinna gives $\tau \leqq q+\pi d$. The other inequalities are self-evident.

Theorem 3 shows that the width of the strip of convergence is affected by the frequency of zeros and poles as well as by the rate of growth of the function in parts of the plane distant from the real axis, since all these factors influence the rate of growth of the function near the real axis. That Theorem 3 is the best of its kind is brought out by the following examples.

We first consider $f(z)=\Gamma(a-i z), a>0$. By Stirling's formula and Theorem 1 we have $\tau=\min (a, \pi / 2)$. Applying Theorem 3 to $\Gamma(a-i z)$ in the half-plane $y>-a$, we find $\beta=+\infty, d=0$, and, again by Stirling's formula, $q=\pi / 2$, so that the inequality $\tau \leqq \min (\alpha, \beta, q+\pi d)=\min (a, \pi / 2)$ becomes exact. We may of course also apply the theorem to an arbitrary lower half-plane. Then $\beta=a, d=1$ and $q=-\pi / 2$, so that the same inequality results.

Our second example is $f(z)=\operatorname{sech} a z, a>0$. Here we can take $\alpha=\beta=\pi /(2 a)$ and find $\pi d=a, q=0$ and $\tau \leqq \min (a, \pi /(2 a))$, which is exact.

\section{REFERENCES} 464.

1. E. Hille, $A$ class of reciprocal functions, Annals of Mathematics, (2), vol. 27 (1926), pp. 427-

2. Contributions to the theory of Hermitian series, Duke Mathematical Journal, vol. 5 (1939), pp. 875-936.

3. R. E. Langer, On the asymptotic solutions of differential equations, with an application to the Bessel functions of large complex order, these Transactions, vol. 34 (1932), pp. 447-480.

4. F. Nevanlinna, Zur Theorie der asymptotischen Potenzreihen, Annales Academiae Scientiarum Fennicae, (A), vol. 12, no. 3 (1918), vii+81 pp.

5. F. and R. Nevanlinna, Über die Eigenschaften analytischer Funktionen in der Umgebung einer singulären Stelle oder Linie, Acta Societatis Scientiarum Fennicae, vol. 50, no. 5 (1922), 46 pp.

6. N. Schwid, The asymptotic forms of the Hermite and Weber functions, these Transactions, vol. 37 (1935), pp. 339-362.

7. G. Szegö, Orthogonal Polynomials, American Mathematical Society Colloquium Publications, vol. 23, New York, 1939, ix $+401 \mathrm{pp}$. 
8. O. Volk, Über die Entwicklung von Funktionen einer komplexen Veränderlichen nach Funktionen, die einer linearen Differentialgleichung zweiter Ordnung genügen, Mathematische Annalen, vol. 86 (1922), pp. 296-316.

9. G. N. Watson, The harmonic functions associated with the parabolic cylinder, Proceedings of the London Mathematical Society, (2), vol. 8 (1910), pp. 393-421.

10. - The harmonic functions associated with the parabolic cylinder, Proceedings of the London Mathematical Society, (2), vol. 17 (1918), pp. 116-148.

11. E. T. Whittaker, On the functions associated with the parabolic cylinder in harmonic analysis. Proceedings of the London Mathematical Society, vol. 35 (1903), pp. 417-427.

12. E. T. Whittaker and G. N. Watson, Modern Analysis, 4th edition, Cambridge, 1927, $\mathrm{x}+608 \mathrm{pp}$.

Yale University,

New Haven, Conn. 